\title{
First-principles investigation of polarization and ion conduction mechanisms in hydroxyapatite
}

\author{
Shusuke Kasamatsu, 1 a) and Osamu Sugino ${ }^{1}$ \\ The Institute for Solid State Physics, the University of Tokyo \\ 5-1-5 Kashiwanoha, Kashiwa-shi, Chiba 27r7-8581, Japan
}

(Dated: 18 December 2017)

\begin{abstract}
We report first-principles simulation of polarization mechanisms in hydroxyapatite to explain the underlying mechanism behind the reported ion conductivities and polarization under electrical poling at elevated temperatures. It is found that ion conduction occurs mainly in the column of $\mathrm{OH}^{-}$ions along the $c$-axis through a combination of the flipping of $\mathrm{OH}^{-}$ions, exchange of proton vacancies between $\mathrm{OH}^{-}$ions, and the hopping of the $\mathrm{OH}^{-}$vacancy. The calculated activation energies are consistent with those found in conductivity measurements and thermally stimulated depolarization current measurements.
\end{abstract}

\section{INTRODUCTION}

Hydroxyapatite $\left[\mathrm{Ca}_{10}\left(\mathrm{PO}_{4}\right)_{6}(\mathrm{OH})_{2}\right](\mathrm{HAp})$ is a wellknown mineral comprising up to $50 \%$ of human bone, and many studies on this material have focused on its use as a biomaterial for, e.g., bone transplants. HAp is also known to exhibit ionic conductivity. Furthermore, nanocrystalline HAp has been shown to exhibit piezo-, pyro-, and ferroelectricity ${ }^{1}$, opening up discussion for possible use of the material in various in vivo and $e x$ vivo energy harvesting and nanoelectronic devices.

On a related but slightly different note, Tanaka and coworkers have reported that this material works remarkably well as an inorganic electret material2 3 . An electret exhibits a permanent dipole and can be understood as an analogue of a magnet with electrical polarization instead of magnetization. By applying DC bias at elevated temperatures and then quenching to room temperature, hydroxyapatite can be poled into electrets. This is explained by the displacement of protons, whose motion at room temperature is frozen due to the relatively high activation energy for diffusion. Thermally stimulated depolarization current (TSDC) spectra shows that in polycrystalline samples, there are up to four polarization mechanisms with different activation energies and relaxation times ${ }^{2 / 4}$. Understanding of the microscopic mechanisms is highly desirable for optimizing this material for electret applications such as micro-energy harvesting and biological tissue engineering.

Figure 1 shows the hexagonal $P 6_{3} / m$ structure of HAp, which is the most frequently encountered structure, especially at elevated temperatures $\sqrt{5 \mid 6}$. The crystal structure consists of columns of hydroxyl $\left(\mathrm{OH}^{-}\right)$ions along the $c$-axis, $\mathrm{PO}_{4}^{3-}$ tetrahedra, and $\mathrm{Ca}^{2+}$ ions. The occupancy of the $\mathrm{O} 4$ and $\mathrm{H}$ sites comprising the $\mathrm{OH}^{-}$ions is $1 / 2$, which may be understood as originating from the orientational and occupational disorder of $\mathrm{OH}^{-}$ions at elevated temperature. That is, the structure along the $c$-axis can be understood as two $\mathrm{OH}$ sites centered at $z=1 / 4$ and

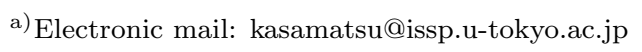
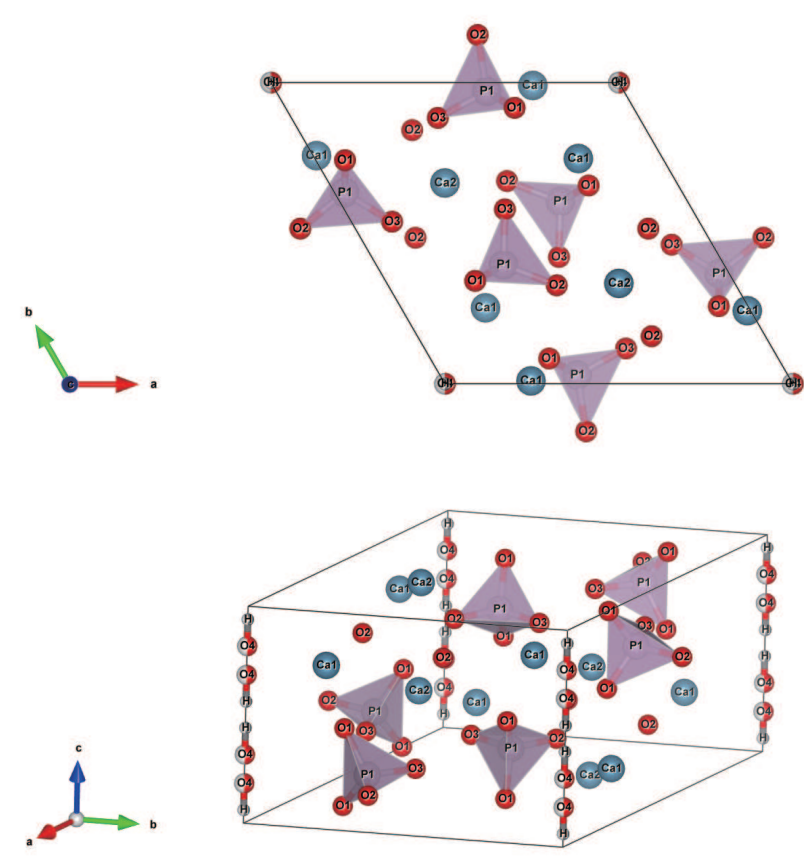

FIG. 1. The $P 6_{3} / m$ crystal structure of hydroxyapatite.

$z=3 / 4$, and $\mathrm{OH}$ ions occupy each site in either the $\mathrm{H}$ up or H-down orientation. Our first-principles simulation results presented below confirm this view. At lower temperatures, there is some discrepancy as to the reported structures, probably owing to different stoichiometries, dopants, defects, disorder, and the intricacies involved in Rietveld fitting of diffraction data ${ }^{1 / 5 / 7 / 8}$. Proposed structures include $P 6_{3}, P 2_{1}, P 2_{1} / b$ and $P 2_{1} / c$, which are all closely related to the $P 6_{3} / m$ structure and differing in slight deviation from the hexagonal angles, slight canting of the $\mathrm{PO}_{4}^{3-}$ tetrahedra, and the ordering of the $\mathrm{OH}^{-}$ direction.

Previously, it was suggested that the ionic conductivity originates from proton hopping between the $\mathrm{OH}^{-}$ ions and the $\mathrm{PO}_{4}^{3-}$ tetrahedra along the $c$-axis based on the aging characteristics of the conductivity 9 . Another work, based on electromotive force and electrol- 
ysis measurements on oxygen concentration cells, concluded that $\mathrm{OH}^{-}$ions are the main conducting species, not protons 10 . A more recent neutron diffraction work claims a sinusoidal proton diffusion pathway along the $c$-axis 6 , but neutron diffraction can only examine probability of finding protons at certain positions and is not a direct proof of long-range diffusion. Moreover, this can account for only one of the four polarization mechanisms noted above. Atomistic simulations should be very useful for such investigation, although there are none, to our knowledge, that focus on ion diffusion in this material. Thus, in this work, we turn to first-principles simulation to investigate possible microscopic mechanisms that can lead to polarization in HAp. The minimum energy pathway and energy barriers for several hypothesized mechanisms are calculated and their relation to various experiments are discussed.

It is well accepted experimentally and theoretically that HAp can accommodate a large number of defects in the $\mathrm{OH}^{-}$column that is introduced through dehydration $9 \mid 11$. That is, one $\mathrm{OH}^{-}$ion and one proton are removed from the $\mathrm{OH}^{-}$column as a water molecule. This leaves one $\mathrm{OH}^{-}$vacancy $\left(\mathrm{V}_{\mathrm{OH}}^{\bullet}\right)$ and one $\mathrm{O}^{2-}$ ion $\left(\mathrm{O}_{\mathrm{OH}}^{\prime}\right)$ at two lattice sites filled originally by $\mathrm{OH}^{-}$ions (Kröger-Vink notation is given in parentheses). $\mathrm{O}_{\mathrm{OH}}^{\prime}$ can also be referred to as a proton vacancy $\left(\mathrm{V}_{\mathrm{H}}^{\prime}\right)$; we use these terms interchangeably depending on whether we are discussing $\mathrm{O}^{2-}$ diffusion or $\mathrm{H}^{+}$diffusion.

It is also known that Ca-deficient HAp can form depending on process conditions ${ }^{12}$; in that case, it was shown through DFT calculations for defect formation energetics that proton interstitials can exist in substantial amounts to satisfy charge neutrality 13 . The interstitial protons are found to be most stable when forming a $\mathrm{H}_{2} \mathrm{O}$ molecule-like structure by binding with $\mathrm{OH}^{-}$ions of the parent lattice. Therefore, we consider the influence of the $\mathrm{OH}^{-}$vacancy $\left(\mathrm{V}_{\mathrm{OH}}^{\bullet}\right)$, proton vacancy $\left(\mathrm{O}_{\mathrm{OH}}^{\prime}\right.$ or $\left.\mathrm{V}_{\mathrm{H}}^{\prime}\right)$, and proton interstitial on the polarization and ion conduction in this material.

\section{METHOD AND MODEL}

The calculations are performed using VASP ${ }^{14 \mid 15}$ code based on the Kohn-Sham formalism of density functional theory (KS-DFT) ${ }^{16 / 17}$. The GGA-PBE parametrization of the exchange-correlation functiona $18 / 19$ is employed. The projector-augmented wave (PAW) method $\sqrt{20}$ is used to describe ion-electron interactions, and the wave functions are expanded by a plane wave basis set with a cutoff energy of $500 \mathrm{eV}$ for lattice-parameter relaxation, and $400 \mathrm{eV}$ for the rest of the calculations. The structural relaxations are performed until forces on each ion become smaller than $10^{-2} \mathrm{eV} / \AA$. First-principles $N V T$ molecular dynamics simulations were carried out with a time step of 0.5 fs using the Nosé thermostat. The minimum energy paths and energy barriers of several candidate polarization/diffusion mechanisms were calculated

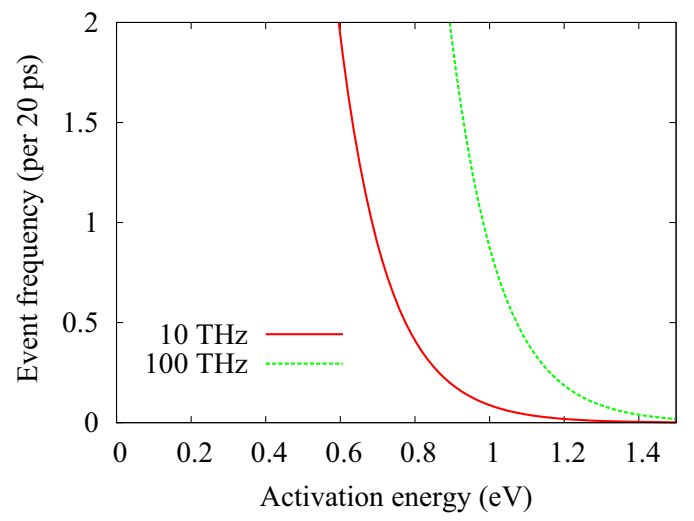

FIG. 2. The event frequency $\gamma=\nu \exp \left(-E_{\mathrm{a}} / k_{\mathrm{B}} T\right)$ at $T=$ $1500 \mathrm{~K}$ for activated processes with a frequency prefactor $\nu$ of $10 \mathrm{THz}$ (solid line) and $100 \mathrm{THz}$ (dashed line) calculated as a function of the activation energy $E_{\mathrm{a}}$.

TABLE I. Comparison of the lattice parameter $a(=b), c$, and fractional coordinate $z$ of the $\mathrm{O}$ and $\mathrm{H}$ atoms in the $\mathrm{OH}$ column of HAp calculated in this work with previous firstprinciples and experimental results.

\begin{tabular}{lcccc}
\hline \hline & $a(=b)(\AA)$ & $c(\AA)$ & $z(\mathrm{O})$ & $z(\mathrm{H})$ \\
\hline This work (GGA-PBE) & 9.552 & 6.912 & 0.276 & 0.418 \\
GGA-PBE (Ref. 11) & 9.536 & 6.904 & 0.286 & 0.428 \\
LDA (Ref. 23) & 9.11 & 6.86 & 0.29 & 0.42 \\
Experiment (Ref. 24) & 9.4302 & 6.8911 & 0.298 & 0.442 \\
Experiment (Ref. 25) & 9.4214 & 6.8814 & - & - \\
\hline \hline
\end{tabular}

using the climbing-image nudged elastic band (CI-NEB) method 21 .

In this work, we consider as the basis structure for our investigation the hexagonal unit cell shown in Fig. 1 . Polarization and ion diffusion mechanisms are investigated using a supercell where the unit cell shown in Fig. 1 is extended by a factor of three in the $c$-axis direction. We note that since we are considering diffusion of ionic species through defects at elevated temperatures, subtle differences in the lattice symmetry mentioned in Sec. I are not very relevant to our work. We consider the possibility of intrinsic proton diffusion in the perfectly stoichiometric HAp. In addition, we consider defectmediated diffusion of proton, hydroxyl, and oxide ions. When a single charged defect is present in the calculation cell, a uniform background charge is introduced to avoid the divergence of the electrostatic energy. Other defects may be examined in the future, although their contribution to the total ionic conductivity and poling under bias should be magnitudes smaller due to higher formation energies, as reported in Ref. 11 and 13 .

\section{RESULTS AND DISCUSSION}

As part of the initial setup for subsequent calculations of diffusion barriers, a simultaneous relaxation of the lat- 

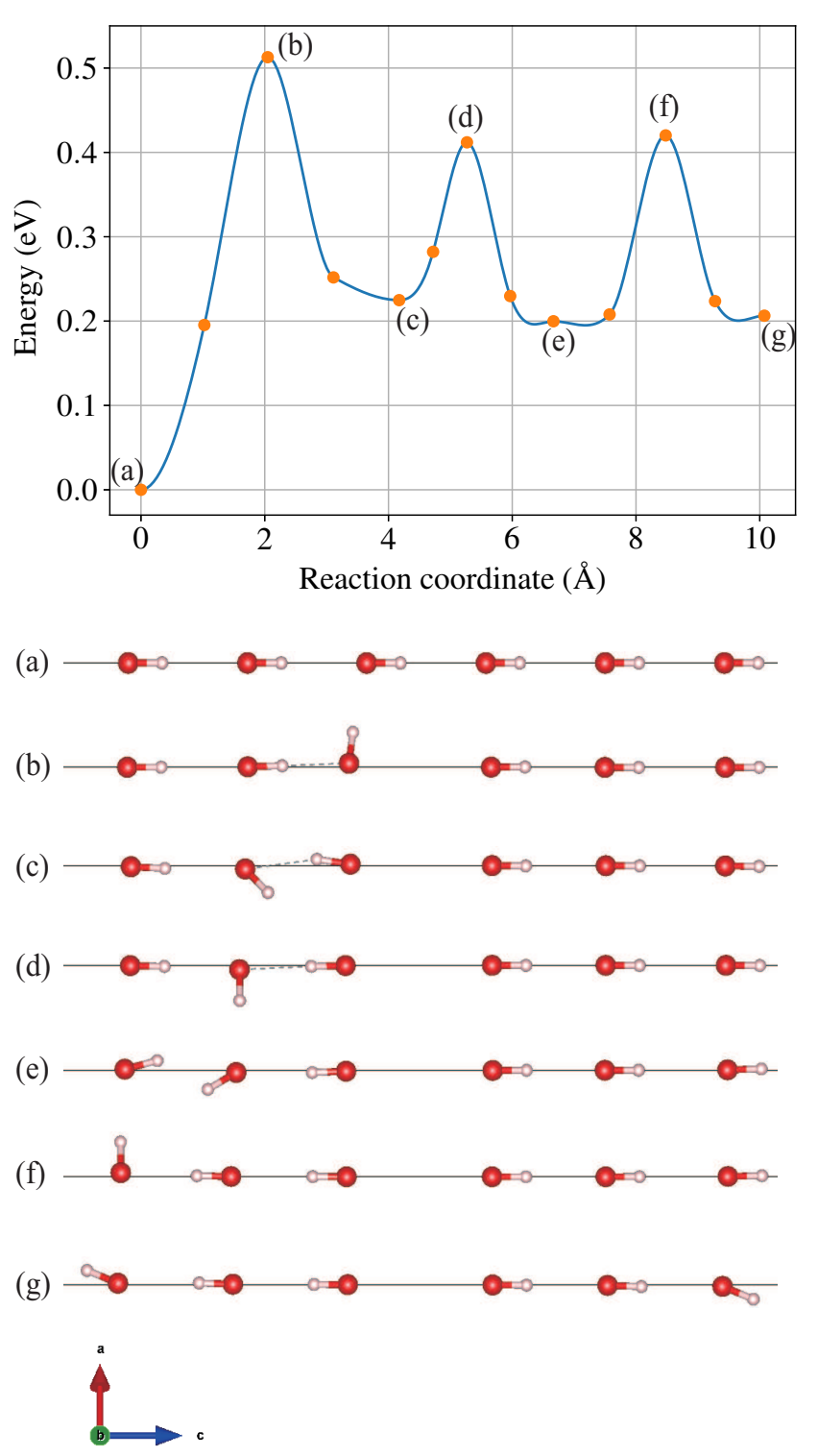

FIG. 3. The minimum energy path for the flipping of $\mathrm{OH}^{-}$ ions from a uniformly polarized configuration (a) to a halfpolarized configuration $(\mathrm{g})$. The structure of the $\mathrm{OH}^{-}$ions (a)-(g) along the minimum energy path are given at the bottom, while their energies along the reaction coordinate are given at the top. Filled circles correspond to the energies of the NEB images used in the calculation, while the lines between the filled circles are obtained through the interpolation scheme of Ref. 22 .

tice and internal parameters was performed on the unit cell of HAp (Fig. 1) where all $\mathrm{OH}^{-}$ions were aligned in the same direction. A $2 \times 2 \times 3 \mathrm{k}$-point mesh centered at the $\Gamma$ point was employed for the Brillouin zone integration. Table 1 compares the calculated lattice parameters with previous theoretical and experimental works. The result is within the typical error range for the GGA-PBE functional when compared to experiment, and is also in good agreement with previous theoretical works employ- ing the same functional. In subsequent calculations, the unit cell was multiplied by a factor of three in the $c$-axis direction and the lattice parameters were kept fixed. We employed a smaller $2 \times 2 \times 1 \mathrm{k}$-point mesh due to the extension of the supercell in the $c$-axis direction.

First, we performed molecular dynamics (MD) simulations at $1,000-1,500 \mathrm{~K}$ for $>20 \mathrm{ps}$ as a quick-anddirty way to find likely polarization and diffusion mechanisms. To estimate the possibility of observing activated processes within the MD simulation time, we calculated event frequencies as a function of activation energy $E_{\mathrm{a}}$ based on harmonic transition state theory ${ }^{26}$ (Fig. 2). Considering the fact that characteristic frequencies of metal oxides are usually of the order of $\sim 10 \mathrm{THz}$, and also considering the fact that we have hydrogen in the system, we can estimate the frequency prefactor $\nu$ entering the rate equation $\gamma=\nu \exp \left(-E_{\mathrm{a}} / k_{\mathrm{B}} T\right)$ to be of the order to 10-100 THz. Thus, from Fig. 2, we can expect to observe processes with $E_{\mathrm{a}}$ of less than $0.6 \mathrm{eV}$ many times within the simulation, while the chances of observing processes with $E_{\mathrm{a}}>1.2 \mathrm{eV}$ are nil.

With the above in mind, we calculated systems with 1) no defects, 2) a proton interstitial, 3) a proton vacancy, and 4) proton vacancy-OH vacancy pair. Flipping of the direction of the $\mathrm{OH}^{-}$ions were observed in all cases, while proton interstitial hopping $(\mathrm{HOH}-\mathrm{OH} \leftrightarrow$ $\mathrm{HO}-\mathrm{HOH}$ ) in the $\mathrm{OH}$ column was observed in 2), $\mathrm{OH}-\mathrm{O}$ $\leftrightarrow$ O-HO proton exchange was observed in 3 ) and 4), and $\mathrm{OH}^{-}$vacancy migration was observed in 4 ). That is, we were able to (luckily) detect migration processes for every defect considered. The fact that they were detected within such short simulations implies that they are also the dominant elementary processes for each ionic specie. Each of these mechanisms is examined in more detail below by using the CI-NEB method.

\section{A. Microscopic mechanisms for polarization and ion diffusion}

\section{OH flip}

As mentioned above, HAp has been shown to be ferroelectric, and the most natural mechanism for polarization reversal would be the flipping of the $\mathrm{OH}^{-}$ions in the $\pm c$ axis directions. Figure 3 shows the minimum energy path for the reversal of the direction of one $\mathrm{OH}^{-}$ion starting from the uniformly polarized system, as well as subsequent flipping of neighboring $\mathrm{OH}^{-}$ions. We find that the flipping of the first $\mathrm{OH}^{-}$ion, which may be considered the nucleation of an opposite polarization domain in the 1D ferroelectric, shows the highest activation energy at $\sim 0.5 \mathrm{eV}$. The activation energy for subsequent enlargement of the domain is lower than $0.25 \mathrm{eV}$. It is interesting to note that when the hydrogen of the $\mathrm{OH}^{-}$ ions face each other, the $\mathrm{OH}^{-}$ions are canted in the $a$ or $b$-axis directions to minimize the repulsion. Also, the total energies of the locally stable structures depend little 
on the "domain size"; the energy is more or less constant when there are two "domain walls" in the unit cell at about $0.2 \mathrm{eV}$ higher than the monodomain state. The relatively low activation energy combined with entropic effects means that the direction of the $\mathrm{OH}^{-}$ions would be highly disordered at elevated temperature. This may explain the 1/2 occupation of the $\mathrm{O} 4$ and $\mathrm{H}$ sites (see Fig. 1) along the $\mathrm{OH}^{-}$columns in the Rietveld fitting of the $\mathrm{P}_{3} / \mathrm{m}$ structure. We also note that the flipping motion of the $\mathrm{OH}^{-}$ions are consistent with proton density mapping based on neutron diffraction measurements 6 .

\section{Formation of intrinsic defects}

In ceramic crystals, ions diffuse through crystals through defects such as vacancies and interstitials, which can be introduced in significant numbers through aliovalent ion doping or in the case of HAp, dehydration as noted above. Although much smaller in number, we may still consider the formation of intrinsic defects which are generated thermally within the material. For example, a proton in one of the $\mathrm{OH}^{-}$ions may be detached and may bond with an adjacent $\mathrm{OH}^{-}(\mathrm{OH}-\mathrm{OH} \leftrightarrow \mathrm{O}-\mathrm{HOH})$, creating what may be considered a proton vacancy-interstitial pair ${ }^{27}$. Other authors have suggested that the $\mathrm{PO}_{4}^{3-}$ tetrahedra around the $\mathrm{OH}^{-}$columns can trap protons ${ }^{249}$. We calculated the energy cost for the former reaction to be $1.9 \mathrm{eV}$ and the latter as $1.8 \mathrm{eV}$, which are too high to explain the experimental results $\$$, where the highest activation energy reported is $\sim 1.3 \mathrm{eV}$. The latter result is also consistent with a previous DFT calculation, which found that a structure where the $\mathrm{O}$ and $\mathrm{H}$ atoms of an $\mathrm{OH}^{-}$ion are split apart is unstable, and the atoms spontaneously recombine upon relaxation to form the $\mathrm{OH}^{-}$ion 28 . In other words, splitting covalent $\mathrm{O}-\mathrm{H}$ bonds costs too much energy without any help from electrostatics (i.e., charge neutrality condition) induced by aliovalent doping or dehydration. Thus, intrinsic defects generated through thermal splitting of $\mathrm{OH}^{-}$have virtually no contribution to the properties of this material.

\section{Proton vacancy}

Next, we consider the role of extrinsic defects introduced through dehydration or charge compensation for cation non-stoichiometries.

First, we consider the migration of proton vacancies. Figure 4 shows one possible chain of events leading to proton vacancy diffusion: flipping of an $\mathrm{OH}^{-}$ion adjacent to a proton vacancy $(-\mathrm{O}-\mathrm{OH}-\leftrightarrow-\mathrm{O}-\mathrm{HO}-)$, followed by exchange of protons between adjacent oxide ions (-O-HO- $\leftrightarrow-\mathrm{OH}-\mathrm{O}-)$. This is basically the proton migration mechanism proposed in Ref. 6] based on neutron diffraction measurements. In some previous works, proton hopping between $\mathrm{OH}^{-}$ions in the $\mathrm{OH}^{-}$column were deemed unviable because the distance between $\mathrm{OH}^{-}$ions

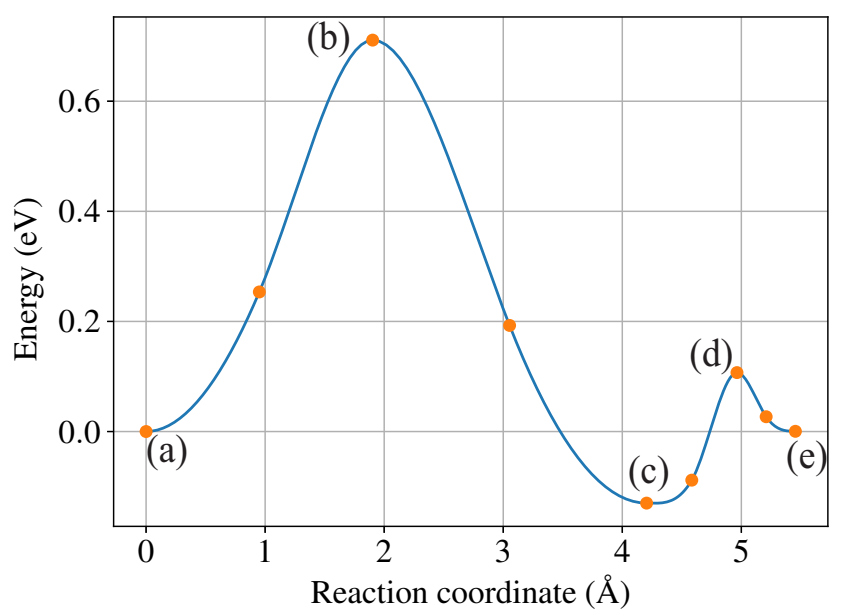

(a)

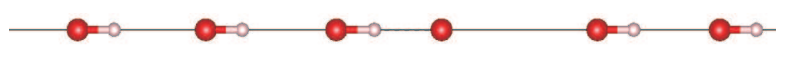

(b)

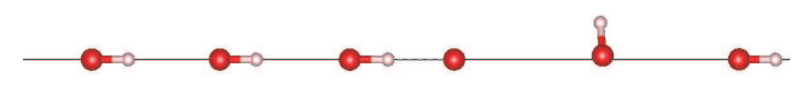

(c)

(d)

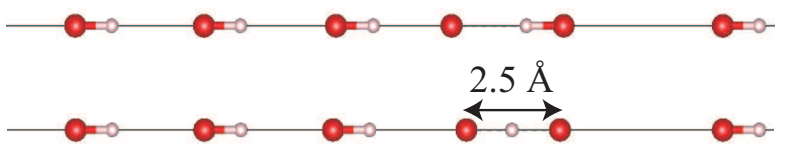

(e)

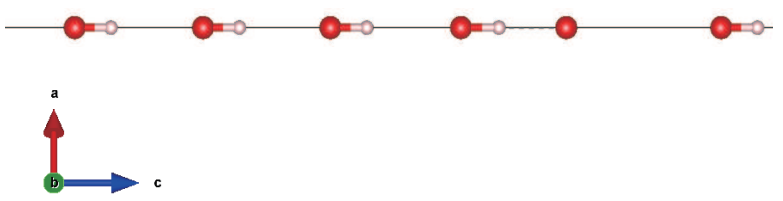

FIG. 4. The minimum energy path for the migration of a proton vacancy. Flipping of an $\mathrm{OH}^{-}$ion next to a proton vacancy (a)-(c) and exchange of protons between adjacent oxide ions (c)-(e) must occur in series for long-range proton vacancy diffusion.

are longer than typical O-O distances in hydrogen bond networks 910 . However, we find that actually, the proton (vacancy) exchange occurs rather easily with an activation energy of $\sim 0.2 \mathrm{eV}[\mathrm{Fig}$ [4(c)-(e)]. This is because as depicted in Fig. 4 (d), $\mathrm{OH}^{-}$ions can vibrate around its stable position without costing too much energy and the $\mathrm{O}-\mathrm{O}$ distance at the transition state is only $2.5 \AA$, which is certainly close enough for the shuttling of protons much like the Grotthus mechanism in the network of water molecules. On the other hand, it is notable that the $\mathrm{OH}^{-}$flipping next to a proton vacancy requires a higher activation energy compared to the pristine nondefective system examined in Fig. 3 .

Figs. 4 (a)-(e) is not the only possible chain of events leading to long range proton migration. For example, the proton vacancy can exchange continuously from right to left without $\mathrm{OH}$ flipping as

$$
-\mathrm{OH}-\mathrm{OH}-\mathrm{O}-\rightarrow-\mathrm{OH}-\mathrm{O}-\mathrm{HO}-\rightarrow-\mathrm{O}-\mathrm{HO}-\mathrm{HO}-
$$




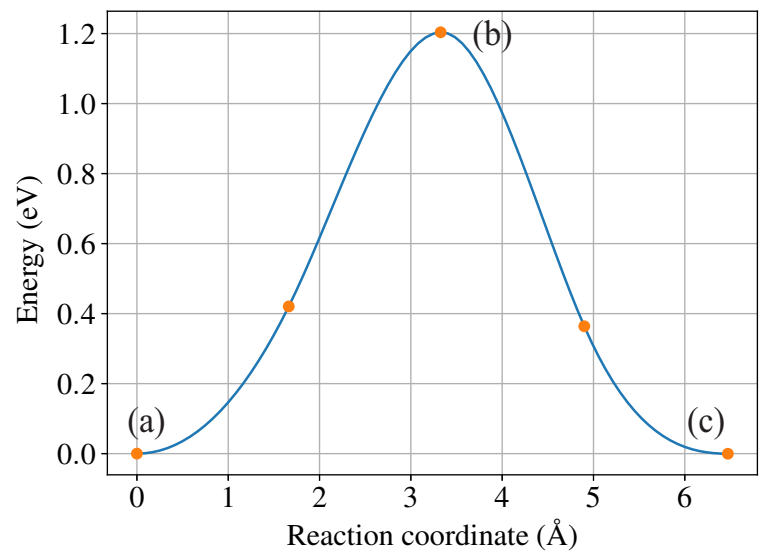

(a)

(b)

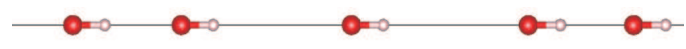

(c)

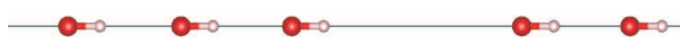

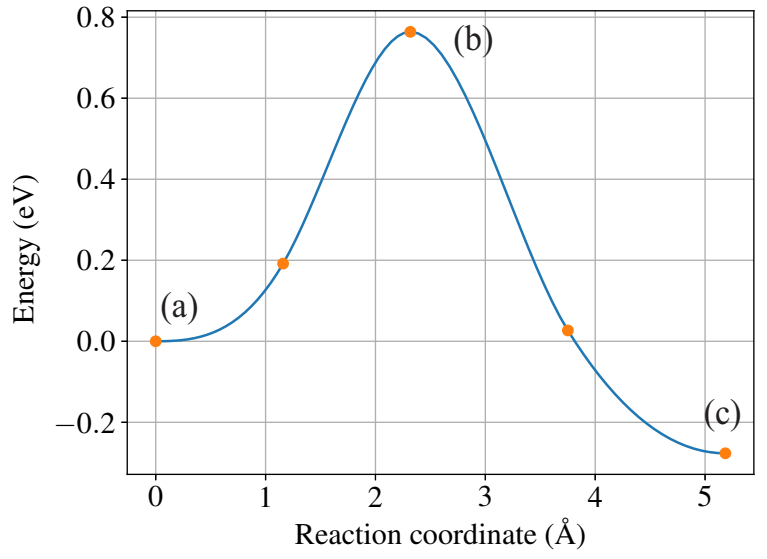

(a)

(b)

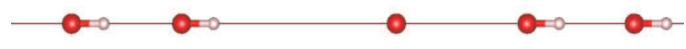

(c)

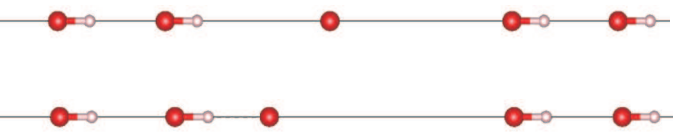

FIG. 5. The minimum energy path for the migration of a $\mathrm{OH}^{-}$vacancy. The left figures depict $\mathrm{V}_{\mathrm{OH}}^{\bullet}-\mathrm{OH}_{\mathrm{OH}}^{\mathrm{x}}$ exchange, while the right figures depict $\mathrm{V}_{\mathrm{OH}}^{\bullet}-\mathrm{O}_{\mathrm{OH}}^{\prime}$ exchange.

We stress, however, that macroscopic polarization involving the motion of more than one proton vacancy cannot occur without $\mathrm{OH}$ flipping. This is easily illustrated if we consider another proton vacancy coming from the right as - $\mathrm{HO}-\mathrm{HO}-\mathrm{O}-$. The $\mathrm{HO}$ immediately to the left of $\mathrm{O}$ must first flip for the proton vacancy to move to the left as

$$
-\mathrm{HO}-\mathrm{HO}-\mathrm{O}-\rightarrow-\mathrm{HO}-\mathrm{OH}-\mathrm{O}-\rightarrow-\mathrm{HO}-\mathrm{O}-\mathrm{HO}-\text {. }
$$

In dehydrated $\mathrm{HAp}, \mathrm{OH}^{-}$vacancies exist in significant amounts in addition to proton vacancies, and this is expected to hinder the diffusion of protons 9 . Indeed, we calculated the energy barrier for the traversal of a proton across an $\mathrm{OH}^{-}$vacancy $\left(-\mathrm{O}-\mathrm{V}_{\mathrm{OH}}-\mathrm{HO}-\leftrightarrow-\mathrm{O}-\mathrm{H}-\mathrm{O}-\right.$ $\left.\leftrightarrow-\mathrm{OH}-\mathrm{V}_{\mathrm{OH}^{-}} \mathrm{O}^{-}\right)$to be higher than $4 \mathrm{eV}$, meaning that such direct proton diffusion across $\mathrm{OH}^{-}$vacancies is unlikely to occur in dehydrated HAp. Thus, we need to consider other mechanisms for long-range proton migration.

\section{4. $\mathrm{OH}^{-}$vacancy}

In the presence of $\mathrm{OH}^{-}$vacancies, it would be logical to consider the possibility of their migration through a vacancy hopping mechanism. If the $\mathrm{OH}^{-}$vacancy hopping occurs at relatively low activation energies, then the protons do not need to traverse across $\mathrm{OH}^{-}$vacancies for long range diffusion. Instead, $\mathrm{OH}^{-}$ions can carry protons through vacancy hopping, or a combination of $\mathrm{OH}^{-}$vacancy hopping, $\mathrm{OH}^{-}$flipping, and $-\mathrm{OH}-\mathrm{O}-\leftrightarrow-\mathrm{O}-\mathrm{HO}-$ proton exchange discussed in preceding sections would allow for long-range ion diffusion. We calculated the $\mathrm{OH}^{-}-\mathrm{V}_{\mathrm{OH}}$ vacancy exchange energy to be $\sim 1.2 \mathrm{eV}$ (Fig. 5 left), and the $\mathrm{OH}^{-}-\mathrm{V}_{\mathrm{OH}}$ vacancy exchange energy to be $\sim 0.8-1.1 \mathrm{eV}$ (Fig. 5 right, depending on the direction of diffusion), when all $\mathrm{OH}$ ions point in the same direction. The variation in the activation energies show that the precise values depend on the polarization of the environment surrounding the migrating ion.

\section{Proton interstitial}

In Ref. 13, it was reported that interstitial protons can be stabilized in low $\mathrm{pH}$ conditions in Ca-deficient HAp, and that the most stable position for the interstitial proton is on the $\mathrm{OH}^{-}$ion, forming an $\mathrm{H}_{2} \mathrm{O}$-molecule like structure. Bonding with $\mathrm{PO}_{4}^{3-}$ with an $\mathrm{O}-\mathrm{H} \cdots \mathrm{O}$ linkage with an adjacent $\mathrm{PO}_{4}^{3-}$ group was also found to be locally stable. These configurations were also realized in our first-principles molecular dynamics simulations mentioned above. Hopping along the $\mathrm{OH}^{-}$column was also 

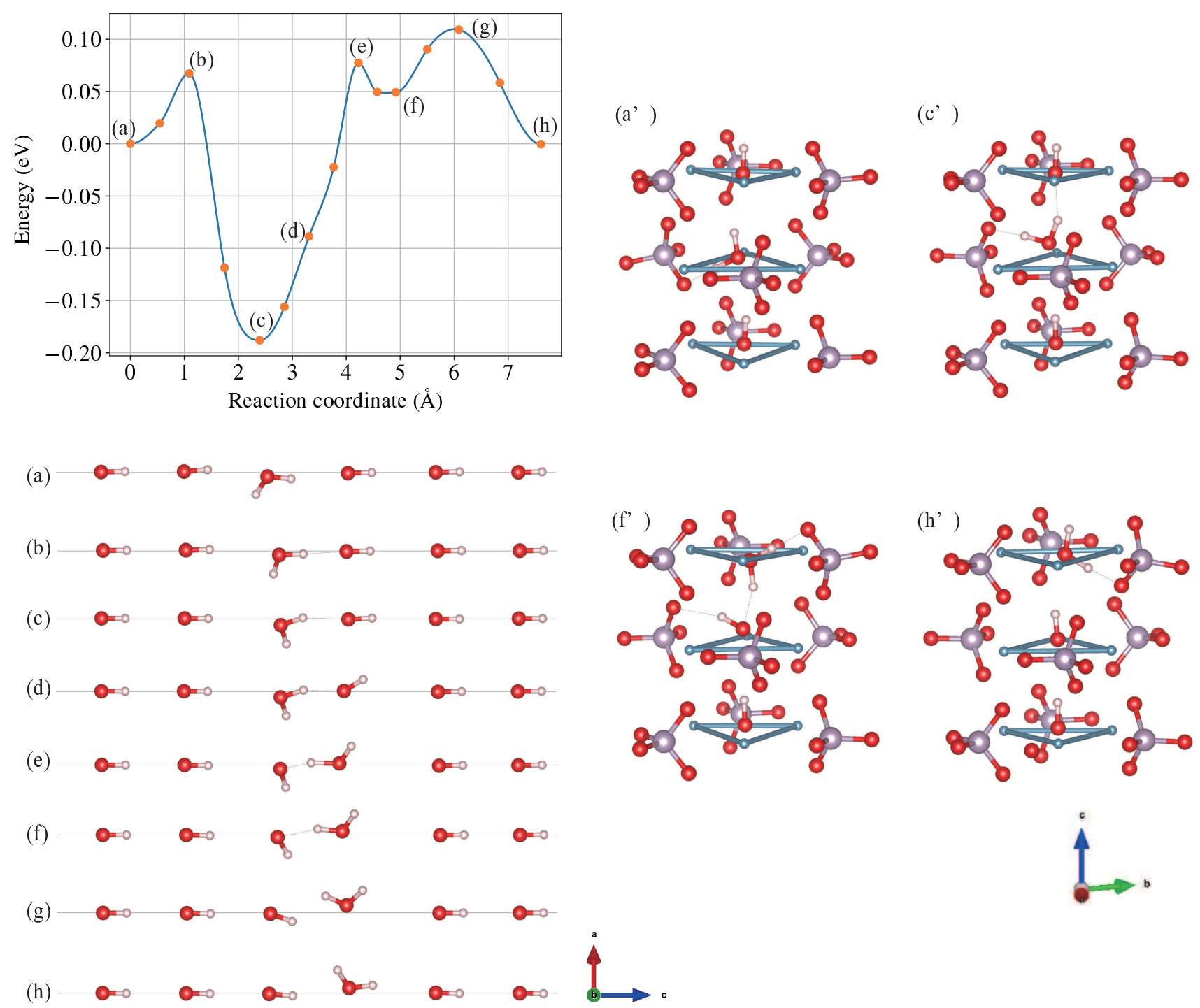

FIG. 6. The minimum energy path for the migration of a proton interstitial within the $\mathrm{OH}^{-}$column. The energy along the path is given at the upper left, while the corresponding structures along the migration path is shown in the lower left and right-side figures.

observed in the molecular dynamics simulation, and the migration pathway for this mechanism calculated using CI-NEB is shown in Fig. 6. The migration proceeds with rotation of $\mathrm{H}_{2} \mathrm{O}$ and $\mathrm{OH}^{-}$combined with proton hopping. The total energy barrier is $\sim 0.3 \mathrm{eV}$, so this is indeed a viable mechanism for proton conduction in Cadeficient HAp. It can be seen from Figures 6 (a')-(h') that hydrogen bonding with neighboring $\mathrm{PO}_{4}^{--}$groups is utilized to stabilize the structures along the minimum energy path. Ref. 13 also showed the hydrogen bonding by examining the defect wave function.

Although we did not observe proton interstitial migration outside of the $\mathrm{OH}^{-}$column in the molecular dynamics simulation (perhaps due to the limited simulation time), we searched possible migration pathways in the $a b$ plane and found the pathway shown in Fig. 7. The en- ergy barrier is $\sim 1.2 \mathrm{eV}$, which is much higher than that within the $\mathrm{OH}^{-}$column. Thus, we can conclude that the interstitial migration is dominated by the $\mathrm{OH}^{-}$column. However, migration in the $a b$ plane may become relevant when bias voltage is applied perpendicular to the $c$-axis during electrical poling of a single crystal sample.

\section{B. Comparison of calculated activation energies with experiment}

Table II tabulates the mechanisms and corresponding activation energies calculated in preceding sections. The lowest activation energy for a single $\mathrm{OH}$ flip is $0.2 \mathrm{eV}$ as shown in Fig. 3 (c-e) and (e-g), which corresponds to the growth of the 1D polarization domain by sequential flip- 

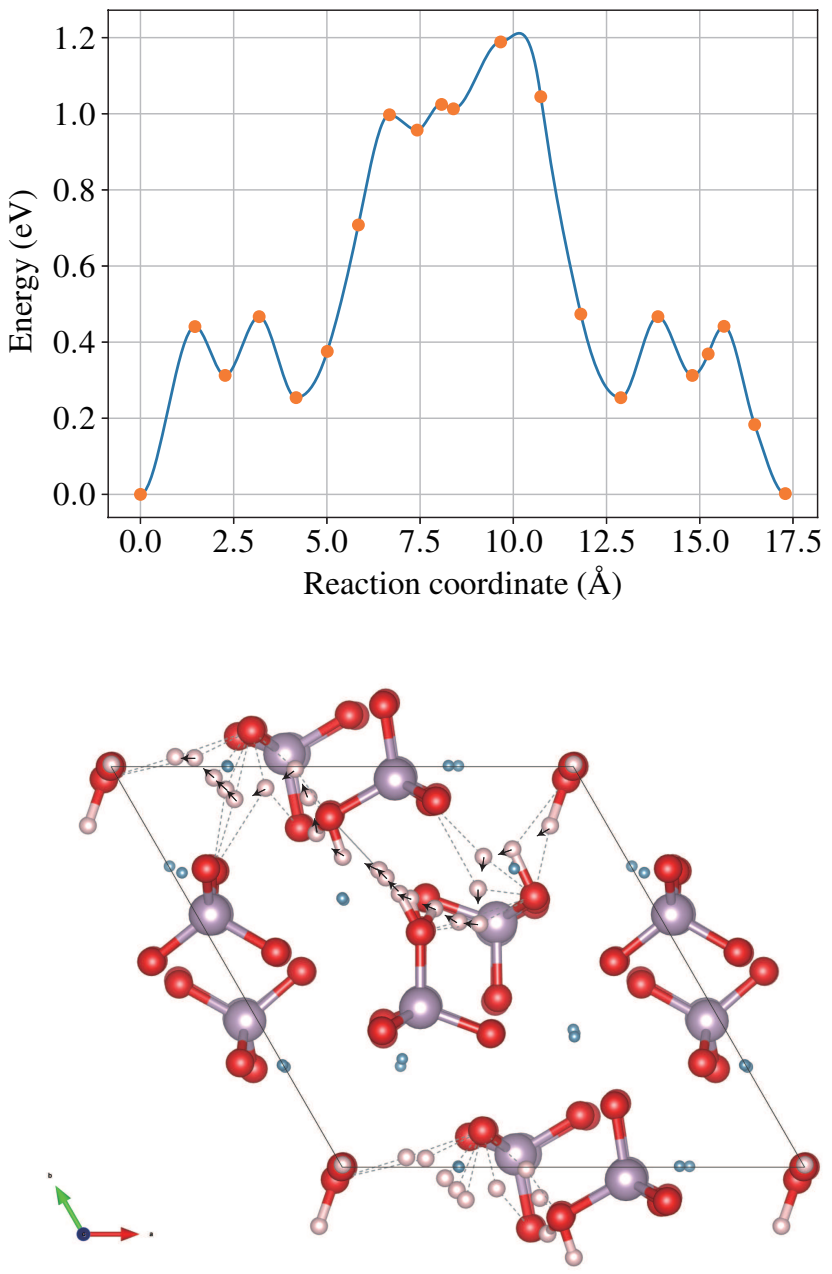

FIG. 7. The minimum energy path for the migration of the proton interstitial. The energy along the pathway is shown in the upper figure, while the migration of the proton interstitial is shown in the bottom. Only the motion of the migrating proton is shown while all other atoms are displayed at their initial positions. Arrows are guides for the eye. A full animation is provided in the online supplement.

ping of adjacent $\mathrm{OH}$ ions. The highest calculated activation energy for $\mathrm{OH}$ flipping is $0.8 \mathrm{eV}$, which corresponds to the flip occurring next to a proton vacancy as in Fig. 4 (c-a). As for $\mathrm{OH}-\mathrm{O} \leftrightarrow \mathrm{O}-\mathrm{HO}$ exchange, the calculated values are $0.2 \mathrm{eV}$ and $0.1 \mathrm{eV}$ in Fig. 4 ( $(\mathrm{c}-\mathrm{e})$ and $(\mathrm{e}-\mathrm{c})$. $\mathrm{OH}$ vacancy hopping occurs with activation energies of

TABLE II. Calculated activation energies $E_{\mathrm{a}}$ of dominant polarization and ion migration mechanisms.

\begin{tabular}{lc}
\hline \hline Mechanism & $E_{\mathrm{a}}$ \\
\hline OH flipping & $0.2-0.8 \mathrm{eV}$ \\
OH-O $\leftrightarrow$ O-HO exchange & $0.1-0.2 \mathrm{eV}$ \\
OH vacancy hopping & $0.8-1.2 \mathrm{eV}$ \\
Interstitial proton hopping & $0.025-0.25 \mathrm{eV}$ \\
\hline \hline
\end{tabular}

0.8-1.2 eV according to Fig. 5, depending on whether the vacancy-ion exchange occurs with $\mathrm{OH}$ or $\mathrm{O}$, and also on the polarization of the environment in the initial and final states. In the case of interstitial proton hopping along the $\mathrm{OH}$ column, there are several local minima. The lowest and highest activation energies are $0.025 \mathrm{eV}$ and $0.25 \mathrm{eV}$ in Fig. 6. $(\mathrm{f}-\mathrm{c})$ and $(\mathrm{c}-\mathrm{f})$ corresponding to the two directions in $\mathrm{HO}-\mathrm{HOH} \leftrightarrow \mathrm{HOH}-\mathrm{OH}$ proton exchange. It should be noted that the calculated values do not incorporate all possible variations due to the environment surrounding the local hopping/flipping site. Since long-range coulombic interactions are prevalent in this ionic material, it would be quite difficult to perform an exhaustive calculation taking into account all possible $\mathrm{OH}$ orientation and defect arrangements around the local hopping/flipping site. However, we can still estimate the effect of the environment from the limited number of varying defect arrangements taken into account in this work, and they suggest that the variations are smaller than few tenths of an eV as in Table II.

The activation energies of the elementary processes calculated in this work do not correspond directly to those obtained from macroscopic conductivity measurements. To perform a direct comparison, one would have to perform e.g., kinetic Monte Carlo simulation to simulate the macroscopic behavior due to the competition and cooperation between microscopic elementary processes. However, microscopic activation energies often do correlate, to a certain degree, to those obtained from the Arrhenius plots of macroscopic measurements [they turn out to be equal according to random walk theory if there is only one mechanism and if the rate of that mechanism does not depend on the configuration (see, e.g., Ref. 29)]. In the following, we attempt to interpret available experimental data based on Table II. We note that the degree of dehydration, and thus the ion carrier concentration, can change during conductivity or depolarization measurements if removal or addition of $\mathrm{H}_{2} \mathrm{O}$ is not blocked. In this case, the dehydration energy would also contribute to the experimentally obtained activation energies. Such effects are usually negligible due to the use of blocking electrodes in electrical measurements if the temperature is kept below $\sim 1000 \mathrm{~K}^{9}$. Therefore, in the following discussion, we assume that generation of thermally activated carriers do not contribute to the measured activation energies.

The literature on conductivity measurements of stoichiometric or partially dehydrated HAp $\frac{9|10| 12|30| 31]}{\text { point }}$ to at least two rate-determining processes depending on the stoichiometry and degree of dehydration; there seems to be one process with an activation energy of $\sim 0.7 \mathrm{eV}$, and another with $\gtrsim 1 \mathrm{eV}$ that becomes more dominant as dehydration proceeds in HAp. By comparing to Table II] the former process may be explained by proton conduction through $\mathrm{OH}^{-}$flipping combined with $-\mathrm{OH}-\mathrm{O}-$ $\leftrightarrow-\mathrm{O}-\mathrm{HO}$ - proton exchange, where the highest activation barrier was calculated to be $\sim 0.8 \mathrm{eV}$. The latter process likely kicks in when many $\mathrm{OH}^{-}$vacancies are in- 
troduced due to dehydration; in this case, $\mathrm{OH}^{-}$vacancy hopping with an activation energy of $\sim 1 \mathrm{eV}$ becomes the rate-determining step in ion conduction along the $\mathrm{OH}$ column. Furthermore, TSDC experiments ${ }^{2 / 4}$ report up to four polarization relaxation processes: one with an activation energy of $\sim 0.4 \mathrm{eV}$, two with $\sim 0.7 \mathrm{eV}$, and another with $\sim 1 \mathrm{eV}$. The latter two activation energies are again very close to those calculated in this work, suggesting that the $\mathrm{OH}^{-}$flipping and $\mathrm{OH}^{-}$vacancy hopping can be considered the rate-determining microscopic mechanisms for at least two of the reported polarization relaxation processes.

On the other hand, Ca-deficient HAp shows a rather complicated temperature dependence in the conductivity 12 due to its instability against dehydration and decomposition to stoichiometric HAp and $\beta$ tricalcium phosphate $\mathrm{Ca}_{3}\left[\mathrm{PO}_{4}\right]_{2}$. An abnormally high conductivity was reported for Ca-deficient HAp whiskers in the temperature range of $250-400{ }^{\circ} \mathrm{C} 12$. Since proton interstitials are expected to exist in significant amounts in Ca-deficient $\mathrm{HAp}^{13}$, such high conductivity may be attributed to the interstitial proton hopping in the $\mathrm{OH}^{-}$ column with a relatively low activation energy of $\sim 0.25$ $\mathrm{eV}$.

For further correlation with experimental data, we would have to consider the effect of the microstructure; for example, existence of trapping/detrapping mechanisms at grain boundaries and proton conduction through interfacial water have been suggested in the literature ${ }^{2}$. Multiscale simulations are necessary for examining polarization mechanisms at longer time and length scales such as formation of space charges ${ }^{32 / 33}$ and their evolution under ac bias34. Such undertakings are beyond the scope of this work, but should be considered for further understanding of this and similar materials systems to be used in various electrical/electrochemical devices.

\section{CONCLUSION}

In this work, we examined polarization and ion diffusion processes in hydroxyapatite using first-principles simulation. We found that the dominant polarization/diffusion mechanisms mainly occur in the column of $\mathrm{OH}^{-}$ions aligned along the $c$-axis, and that the main processes are the flipping of the direction of $\mathrm{OH}^{-}$ions, exchange of protons between oxide ions $(-\mathrm{OH}-\mathrm{O}-\leftrightarrow-$ $\mathrm{O}-\mathrm{HO}-$ ), and the hopping of $\mathrm{OH}^{-}$vacancies. When proton interstitials are present as in the case of Ca-deficient HAp, interstitial proton hopping occurs mainly on the $\mathrm{OH}^{-}$column, although diffusion in the $a b$ plane is also viable under bias. The calculated activation energies are consistent with conductivity and TSDC measurements.

\section{ACKNOWLEDGMENTS}

The authors thank Yumi Tanaka of the Tokyo University of Science for fruitful discussion. The calculations were performed on the SGI ICE XA system at Institute for Solid State Physics, the University of Tokyo. This work was supported by CREST, Japan Science and Technology Agency. S. K. is also supported by Grant-inAid for Young Scientists (B) (No. 15K20953) by Japan Society for the Promotion of Science. Atomic structure figures were created using the visualization software VESTA ${ }^{35}$.

${ }^{1}$ S. B. Lang, S. A. M. Tofail, A. L. Kholkin, M. Wojtaś, M. Gregor, A. A. Gandhi, Y. Wang, S. Bauer, M. Krause, and A. Plecenik, Sci. Rep. 3, 2215 (2013)

${ }^{2}$ Y. Tanaka, T. Iwasaki, M. Nakamura, A. Nagai, K. Katayama, and K. Yamashita, J. Appl. Phys. 107, 014107 (2010)

${ }^{3}$ K. Yamashita, N. Oikawa, and T. Umegaki, Chem. Mater. 8, 2697 (1996)

${ }^{4}$ N. Horiuchi, M. Nakamura, A. Nagai, K. Katayama, and K. Yamashita, J. Appl. Phys. 112, 074901 (2012).

${ }^{5}$ G. Ma and X. Y. Liu, Cryst. Growth Des. 9, 2991 (2009)

${ }^{6}$ M. Yashima, N. Kubo, K. Omoto, H. Fujimori, K. Fujii, and K. Ohoyama, J. Phys. Chem. C 118, 5180 (2014).

${ }^{7}$ J. C. Elliott, P. E. Mackie, and R. A. Young, Science 180, 1055 (1973)

${ }^{\gamma}$ D. Haverty, S. A. M. Tofail, K. T. Stanton, and J. B. McMonagle, Phys. Rev. B 71, 094103 (2005)

${ }^{9}$ K. Yamashita, J. Am. Ceram. Soc. 78, 1191 (1995)

${ }^{10}$ T. Takahashi, S. Tanase, and O. Yamamoto, Electrochim. Acta 23, 369 (1978).

${ }^{11} \mathrm{~K}$. Matsunaga and A. Kuwabara, Phys. Rev. B 75, 014102 (2007)

${ }^{12}$ Y. Tanaka, M. Nakamura, A. Nagai, T. Toyama, and K. Yamashita, Mater. Sci. Eng., B 161, 115 (2009)

${ }^{13}$ K. Matsunaga, Phys. Rev. B 77, 104106 (2008).

${ }^{14}$ G. Kresse and J. Furthmüller, Phys. Rev. B 54, 11169 (1996).

${ }^{15} \mathrm{G}$. Kresse and J. Furthmüller, Comp. Mater. Sci. 6, 15 (1996).

${ }^{16} \mathrm{P}$. Hohenberg and W. Kohn, Phys. Rev. 136, B864 (1964).

${ }^{17}$ W. Kohn and L. J. Sham, Phys. Rev. 140, A1133 (1965).

${ }^{18}$ J. P. Perdew, K. Burke, and M. Ernzerhof, Phys. Rev. Lett. 77, 3865 (1996)

${ }^{19}$ J. P. Perdew, K. Burke, and M. Ernzerhof, Phys. Rev. Lett. 78, 1396 (1997)

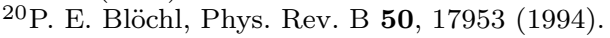

${ }^{21}$ G. Henkelman, B. P. Uberuaga, and H. Jonsson, J. Chem. Phys. 113, 9901 (2000)

${ }^{22}$ G. Henkelman and H. Jónsson, J. Chem. Phys. 113, 9978 (2000)

${ }^{23}$ L. Calderín, M. J. Stott, and A. Rubio, Phys. Rev. B 67, 134106 (2003)

${ }^{24}$ J. Y. Kim, R. R. Fenton, B. A. Hunter, and B. J. Kennedy, Aust. J. Chem. 53, 679 (2000)

${ }^{25}$ P. Alberius-Henning, E. Adolfsson, J. Grins, and A. Fitch, J. Mater. Sci. 36, 663 (2001)

${ }^{26}$ G. H. Vineyard, J. Phys. Chem. Solids 3, 121 (1957).

${ }^{27}$ C. M. Gobinda and F. Freund, J. Chem. Soc., Dalton Trans. 0, 949 (1981).

${ }^{28}$ N. de Leeuw, Phys. Chem. Chem. Phys., 4, 3865 (2002).

${ }^{29} \mathrm{P}$. Shewmon, Diffusion in solids (The Minerals, Metals, \& Materials Society, 1989).

${ }^{30}$ A. Bouhaouss, A. Laghzizil, A. Bensaoud, M. Ferhat, G. Lorent, and J. Livage, Int. J. Inorg. Mater. 3, 743 (2001)

${ }^{31}$ Y. Tanaka, M. Kikuchi, K. Tanaka, K. Hashimoto, J. Hojo, M. Nakamura, A. Nagai, T. Sugiyama, F. Munakata, and K. Yamashita, J. Am. Ceram. Soc. 93, 3577 (2010)

${ }^{32}$ S. Kasamatsu, T. Tada, and S. Watanabe, Solid State Ionics 183, $20(2011)$ 
${ }^{33}$ S. Kasamatsu, T. Tada, and S. Watanabe, Solid State Ionics 226, $62(2012)$
${ }^{34}$ R. Pornprasertsuk, J. Cheng, H. Huang, and F. B. Prinz, Solid State Ionics 178, 195 (2007).

${ }^{35}$ K. Momma and F. Izumi, J. Appl. Crystallogr. 41, 653 (2008). 